\title{
On the Present Time Approximate Values of the Characteristic Parameters of the Primordial Gravitational Waves
}

\author{
M. Melek \\ Astronomy Department, Faculty of Science, Cairo University, Orman, \\ Giza,Egypt; melek@frcu.eun.eg
}

\begin{abstract}
.
An approach is used to find out the present time approximate values of the characteristic amplitudes, the spectral densities, the intensties and the frequencies of the Primordial Gravitational Waves (PmGW), in the spatially perturbed Friedman - Robertson - Walker (FRW) adiabatically expanding Universe. The temporal variation of the magnitude of the time gradient of the characteristic amplitudes of the PmGW is calculated in spatial perturbed FRW Universe. The obtained results via this non-conventional approach may be of interest to shed some light on the possibilities of searching for the PmGW at definite frequency bands by the existing and the future networks of detectors.
\end{abstract}

\section{Introduction}

The Big Bang can be considered as the strongest explosion that had ever happened in the Universe. Since much weaker explosions; e.g. supernovae; under certain conditions do produce gravitational waves (GW) with definite amplitudes and frequencies, then it is reasonable to believe that the Big Bang had produced GW which is called primordial GW (PmGW) [cf. Maggiore (2000); Lyth (1999) and Kolb (1990)]. The uncertainties in the cosmological mechanisms producing those PmGW lead to uncertainties in their theoretical predictions. Therefore, it is needed to use general assumptions and procedures that can help in characterizing the PmGW. If one supposes that the PmGW are isotropic and unpolarized, then their main properties are their amplitudes and their frequency spectrum [cf. Astone, et al. (2000)]. This spectrum can be characterized by three related quantities [cf. Maggoire (2000)] 1. the intensity of $\mathrm{GW} ; \mathbf{I}_{g w}(f) ; 2$. the characteristic amplitude; $\mathbf{A}(f, t)$; and 3 . the spectral density; $\mathbf{D}(f)$. The characteristic parameters of the PmGW are explicit functions of their frequencies which are taken to be time dependent through the Universe's evolution [Maggiore (2000)]. Therefore, the characteristic amplitudes and frequencies of the PmGW can be taken; as functions; as follows $\mathbf{A}=\mathbf{A}(f(t), t)$ and $f=f(t)$, for the purpose of investigating their behaviour since their production time $\left(t_{\text {pro. }}\right)$ passing by the matter formation time $\left(t_{m}\right)$ till the present time $\left(t_{0}\right)$ in the spatially perturbed FRW expanding Universe. Our non-conventional procedure to do that is to define the magnitude of the gradient of the characteristic amplitudes of the PmGW existing in our expanding Universe, which is taken to be the spatially 
perturbed FRW Universe. The magnitude of the gradient of the amplitude of the PmGW is defined as follows:

$$
M=\left(g^{\mu \nu} \mathbf{A}_{\mu} \mathbf{A}_{\nu}\right)^{\frac{1}{2}},
$$

where $g^{\mu \nu}$ is the contravariant metric tensor of the spatially perturbed FRW Universe, which its line element can be expressed as follows [cf. Maggiore (2000) and Allen (1996) and references therein]:

$$
d S^{2}=R^{2}(t)\left\{d t^{2}-\frac{1+\delta_{11}}{1-k r^{2}} d r^{2}-\delta_{12} r d r d \Omega-r^{2}\left(1+\delta_{22}\right) d \Omega^{2}\right\}
$$

$R(t)$ is the scale factor; $\delta_{11}, \delta_{12}, \delta_{22}$ are the spatial perturbations; $\mathbf{A}_{\mu}=\frac{\partial \mathbf{A}}{\partial x^{\mu}}$ is the time- like covariant gradient vector; $\Omega$ is a solid angle defined in terms of $\theta, \phi$ as follows: $d \Omega^{2}=d \theta^{2}+\sin ^{2} \theta d \phi^{2}$ and $\mu, \nu=0,1,2$. It is worth to point out here that the neglection of the time perturbations in the metric (5) can be understood as a kind of statistical average of the perturbations in the Universe through its evolution [cf. Melek $(2002,2000)$ and references therein]. Hence, it is natural to define a covariant function $V$ which measures the temporal variation of the magnitude of the gradient of $\mathbf{A}$ as follows:

$$
V=\frac{d}{d S}\left(g^{\mu \nu} \mathbf{A}_{\mu} \mathbf{A}_{\nu}\right)^{\frac{1}{2}}
$$

where $S$ is the proper time. It is possible to prove that the function $V$ can be expressed as follows [cf. Melek (2002, 2000) and references therein]:

$$
V=\frac{1}{M} g^{\mu \nu} \mathbf{A}_{\mu ; \sigma} \mathbf{A}_{\nu} U^{\sigma}
$$

where $\mathbf{A}_{\mu ; \sigma}$ is the covariant derivative of $\mathbf{A}_{\mu}$ with respect to $x^{\sigma}$ and $U^{\sigma}=\frac{d x^{\sigma}}{d S}$.

Since the evolution of the primordial cosmic quantities is driven by the Universe's expansion and its effect on them. Therefore, it is of interest to use the function $V$ to figure out the influence of the Universe's expansion on the time variation of the characteristic amplitudes of the PmGW.

\section{The Characteristic Parameters Of PmGW}

Using the metric (2) and carrying out the calculations of the function $V$ in a spatial comoving coordinates (i.e. $\frac{d r}{d S}=\frac{d \Omega}{d S}=0$ ), the expression (4) will read:

$$
V_{a m p .}=\frac{U^{0}}{R(t)}\left\{\frac{\partial}{\partial t}\left(\frac{\partial \mathbf{A}}{\partial t}\right)-H(t)\left(\frac{\partial \mathbf{A}}{\partial t}\right)\right\}
$$

where $H(t)=\frac{1}{R(t)} \frac{d R(t)}{d t}$ is the Hubble parameter. This expression gives the temporal change of the magnitude of the time gradient of the characteristic amplitudes of the PmGW in the FRW expanding Universe.

If the universe expands adiabatically, then it is reasonable to assume that the amplitudes and the frequencies of the PmGW are decreasing as the scale factor $R(t)$ is increasing; following these relations [cf. Maggiore (2000)]:

$$
\mathbf{A}(f(t), t) R(t)=\text { Constant }
$$




$$
f(t) R(t)=\text { Constant. }
$$

It is clear from (6), that the time gradient of the amplitude $\mathbf{A}(f(t), t)$ decreases as $R^{-2}(t)$ which means that the amplitude decreases with respect to the time $t$, then the fuction $V$; which is defined by (3)and can be expressed as $V=$ $\frac{d}{d S}\left(\frac{A_{0}}{R(t)}\right)=\frac{\partial}{\partial t}\left(\frac{A_{0}}{R(t)}\right) U^{0}$; should be negative. Since $U^{0}$ and $R(t)$ are positive functions, then as a direct consequence of negative $V$ which is given by (5), one obtains the following differential inequality:

$$
\frac{\partial}{\partial t}\left(\frac{\partial \mathbf{A}}{\partial t}\right)<H(t) \frac{\partial \mathbf{A}}{\partial t} .
$$

Now we shall proceed to see how can we make use of this result to figure out the explicit dependence of the amplitudes of the PmGW on their frequencies and the time through the Hubble parameter. Using (6) and (7) and their time derivatives; one can obtain the values of $\frac{\partial \mathbf{A}}{\partial t}$ and $\frac{\partial}{\partial t}\left(\frac{\partial \mathbf{A}}{\partial t}\right)$ which can be inserted in (8) to lead to the following partial differential inequality for the amplitude of the PmGW:

$$
f^{2} \frac{\partial}{\partial f}\left(\frac{\partial \mathbf{A}}{\partial f}\right)+\left(3-\frac{1}{H^{2}} \frac{d H}{d t}\right) f \frac{\partial \mathbf{A}}{\partial f}-\left(2-\frac{1}{H^{2}} \frac{d H}{d t}\right) \mathbf{A}>0 .
$$

It is possible to prove that the amplitude satisfying (9); during the radiation and matter eras (i.e. $t_{\text {pro. }} \leq t \leq t_{m}$ and $t_{m} \leq t \leq t_{0}$ ); is given, respectively, as:

$$
\begin{gathered}
\mathbf{A}(f(t), t)<\mathbf{A}_{\text {pro. }}\left(\frac{f}{f_{\text {pro. }}}\right)^{a_{1}(t)}, \\
\mathbf{A}(f(t), t)<\mathbf{A}_{\text {mat. }}\left(\frac{f}{f_{\text {mat. }}}\right)^{a_{1}(t)}
\end{gathered}
$$

where: $\mathbf{A}_{\text {pro. }} \mathbf{A}_{\text {mat }}$. are the amplitudes at the PmGW's production and atoms formation times; respectively;

$$
\left.a_{1}(t)<-\left(1-\frac{1}{2 H^{2}} \frac{d H}{d t}\right)+\left[3-\frac{1}{2 H^{2}} \frac{d H}{d t}\right)^{2}\right]^{\frac{1}{2}}\left[1-\frac{1}{2 H^{2}} \frac{d H}{d t}\right]^{\frac{1}{2}}
$$

Since the scale factor $R(t)$ is proportional to $t^{\frac{1}{2}}$, during the radiation era, then, $a_{1}$ has the following values: $a_{1}=-2+2 \sqrt{2}$. Hence, the amplitude of the PmGW; during the radiation era; should satisfy the following inequality:

$$
\mathbf{A}<\mathbf{A}_{\text {pro. }}\left(\frac{f}{f_{\text {pro. }}}\right)^{-2+2 \sqrt{2}}
$$

or as:

$$
\mathbf{A}<\mathbf{A}_{\text {pro. }}\left(\frac{t_{\text {pro. }}}{t}\right)^{-1+\sqrt{2}}
$$

In the view of the relation between $\mathbf{A}$ and $\mathbf{D}$ of the PmGW and (13), one can prove that the spectral density of the PmGW; during the radiation era; should satisfy the following inequality:

$$
\mathbf{D}(f)<(\mathbf{D}(\mathbf{f}))_{\text {pro. }}\left(\frac{f}{f_{\text {pro. }}}\right)^{-3+4 \sqrt{2}} .
$$


Also, using the relation between $\mathbf{A}$ and $\mathbf{I}_{g w}$, it is possible to prove that the intensity of the PmGW; during the radiation era; should satisfy the following inequality:

$$
\mathbf{I}_{g w}(f)<\left(\mathbf{I}_{g w .}(f)\right)_{\text {pro. }}\left(\frac{f}{f_{\text {pro. }}}\right)^{-2+4 \sqrt{2}} .
$$

While the scale factor $R(t)$ is proportional to $t^{\frac{2}{3}}$, during the matter era, then $a_{1}(t)$ has the following value: $a_{1}=\frac{-7+\sqrt{105}}{4}$. Hence, the amplitude of the PmGW; during the matter era; should satisfy the following inequality:

$$
\mathbf{A}<\mathbf{A}_{\text {mat. }}\left(\frac{f}{f_{\text {mat. }}}\right)^{\frac{-7+\sqrt{105}}{4}} .
$$

In the view of the relation between $\mathbf{A}$ and $\mathbf{D}$ and (17), one can prove that the spectral density of the PmGW; during the matter era; can be expressed as follows:

$$
\mathbf{D}(f)<(\mathbf{D}(\mathbf{f}))_{m a t} .\left(\frac{f}{f_{\text {mat. }}}\right)^{\frac{-9+\sqrt{105}}{2}} .
$$

Also, using the relation between $\mathbf{A}$ and $\mathbf{I}$ and (18), it is possible to prove that the intensity of the PmGW; during the matter era; is given as follows:

$$
\mathbf{I}_{g w}(f)<\left(\mathbf{I}_{g w .}(f)\right)_{m a t .}\left(\frac{f}{f_{m a t .}}\right)^{\frac{-5+\sqrt{105}}{2}} .
$$

\section{Concluding Remarks}

1. It is of interest to calculate the values of the characteristic amplitudes, the spectral density and the intensity of the PmGW at the matter (atoms) formation time $\left(t_{m}\right)$ as well as at the present time $\left(t_{0}\right)$ in terms of their primordial values. The answer of this question will shed a bit of light on the fesibility of detecting the PmGW by the existing and the future planned networks of detectors. Since the production time of the PmGW is ranging between $10^{-20}$ to $10^{-50.5}$ years [cf. Maggiore (2000); Ciufolini (2001) and references therein] and the Universe's radiation era can be taken to be of order $10^{5}$ years; then the amplitudes, the spectral densities and the intensities of the PmGW; at the matter formation time; are, respectivly, ranging between the following values:

$$
\begin{aligned}
\mathbf{A}_{\text {mat. }}<\left(10^{-10}\right) \mathbf{A}_{\text {pro. }} ; & \mathbf{A}_{\text {mat. }}<\left(10^{-22}\right) \mathbf{A}_{\text {pro. }} . \\
\mathbf{D}_{\text {mat. }}<\left(10^{-32}\right) \mathbf{D}_{\text {pro. }} ; & \mathbf{D}_{\text {mat. }}<\left(10^{-72}\right) \mathbf{D}_{\text {pro. }} . \\
\left(\mathbf{I}_{\text {gw. }}\right)_{\text {mat. }}<\left(10^{-45}\right)\left(\mathbf{I}_{\text {gw. }}\right)_{\text {pro. }} ; & \left(\mathbf{I}_{\text {gw. }}\right)_{\text {mat. }}<\left(10^{-101}\right)\left(\mathbf{I}_{\text {gw. }}\right)_{\text {pro. }} .
\end{aligned}
$$

Also, the matter era can be taken to be of order $10^{10}\left(1-10^{-5}\right)$. Therefore, the amplitudes, the spectral densities and the intensities of the PmGW; at the present time; are, respectively, given by the following approximate values:

$$
\begin{aligned}
& \mathbf{A}_{t_{0}}<\left(10^{-3}\right) \mathbf{A}_{\text {mat. }}, \\
& \mathbf{D}_{t_{0}}<\left(10^{-2}\right) \mathbf{D}_{\text {mat. }},
\end{aligned}
$$




$$
\left(\mathbf{I}_{g w} .\right)_{t_{0}}<\left(10^{-9}\right)\left(\mathbf{I}_{g w} .\right)_{m a t} .
$$

Hence, the values of the amplitudes, the spectral densities and the intensities of the PmGW at the present time; are ranging between the following values in terms of their values at the PmGW's production time:

$$
\begin{aligned}
\mathbf{A}_{t_{0}}<\left(10^{-13}\right) \mathbf{A}_{\text {pro. }} ; & \mathbf{A}_{t_{0}}<\left(10^{-25}\right) \mathbf{A}_{\text {pro. }}, \\
\mathbf{D}_{t_{0}}<\left(10^{-34}\right) \mathbf{D}_{\text {pro. }} ; & \mathbf{D}_{t_{0}} \approx\left(10^{-74}\right) \mathbf{D}_{\text {pro. }}, \\
\left(\mathbf{I}_{\text {gw. }}\right)_{t_{0}}<\left(10^{-54}\right)\left(\mathbf{I}_{\text {gw }}\right)_{\text {pro. }} ; & \left(\mathbf{I}_{\text {gw. }}\right)_{t_{0}}<\left(10^{-110}\right)\left(\mathbf{I}_{\text {gw. }}\right)_{\text {pro. }} .
\end{aligned}
$$

Since the values of the amplitudes, the spectral densities and the intensities of the PmGW at the production time are dependent on their production mechanism [cf. Maggiore (2000); Lyth (1999) and Ciufolini (2001)], which is out of the realm of this paper, then the relations (20), (21), (22), (26), (27) and (28) will give a rough estimate about the order of magnitude of the charateristic parameters of the PmGW at the matter formation time and at the present time for each production mechanism.

2. Using the relation (7) during the matter era in which the scale factor $R(t) \sim t^{\frac{2}{3}}$, which gives the present time frequencies in terms of their values at the production time as; $f\left(t_{0}\right)=f\left(t_{\text {pro. }}\right)\left[\frac{t_{\text {pro. }}}{t_{m}}\right]^{\frac{1}{2}}\left[\frac{t_{m}}{t_{0}}\right]^{\frac{2}{3}}$. Hence, the present time frequencies of the PmGW are ranging between the following values; respectively;

$$
f\left(t_{0}\right) \approx\left(10^{-13}\right) f\left(t_{\text {pro. }}\right) ; \quad f\left(t_{0}\right) \approx\left(10^{-28}\right) f\left(t_{\text {pro. }}\right),
$$

According to relations (26) and (29); it is clear that the detection of the PmGW are; still; questionable either by the existing detectors or by the future planned ones as single detectors, or even by using two detectors [cf. Maggiore (2000); Astone et al. (2000, 1994); Melek (2002a) and Vecchio (2002)].

3. If the PmGW will be detected; in the future; then the relations (29) can be used to calculate their frequencies at their production time as well as its approximate production time, in the spatially perturbed adiabatically expanding FRW Universe.

4. The author believes that this approach can be useful if it will be applied; as well; in the case of the primordial neutrinos, which was decoupled from the primordial plasma during the weak interaction epoch. This will give an idea about the cosmic neutrinos background and the possibility of distinguishing them; via their frequencies and amplitudes; from those of astrophysical origin and the feasibility of detecting them by the existing neutrinos detectors. This is, exactly, what happend in the case of predicting and detecting the microwave background radiation.

\section{References}

Allen B. 1996, in "Les Houches School On Astrophysical Sources Of Gravitational Waves", eds. Marck J.A. and Lasota J.P. (Cambridge University Press)

Astone P., Ferrari V., Maggiore M., \& Romano J. D., 2000, Int. J. Mod. Phys. 9, 361 
Astone P., Lobo J. A., \& Schutz B. F. 1994, CQG, 11, 2093

Ciufolini I., Gorini V., \& Moschella U. 2001, Gravitational Waves, (IoP Publishing Ltd., Bristol and Philadelphia), chapters 12 and 13

Kolb E. W. \& Turner M. S. 1990, The Early Universe (Addison Wesley).

Lyth D. H. \& Riotto A., 1999, Phys. Rep. 314, 1

Maggiore M. 2000, Phys. Rep. 331, 284

Melek M. 2000, Ap\&SS, 272, 417

Melek M. 2002a, CQG, 19, 1343

Melek M. 2002b, Ap\&SS, 281, 4, 743

Vecchio A. 2002, CQG, 19, 1449 